\title{
Representations of $U(2 \infty)$ and the Value of the Fine Structure Constant
}

\author{
William H. KLINK
}

Department of Physics and Astronomy, University of Iowa, Iowa City, Iowa, USA

E-mail: william-klink@uiowa.edu

Received September 28, 2005, in final form December 17, 2005; Published online December 25,2005

Original article is available at http://www.emis.de/journals/SIGMA/2005/Paper028/

\begin{abstract}
A relativistic quantum mechanics is formulated in which all of the interactions are in the four-momentum operator and Lorentz transformations are kinematic. Interactions are introduced through vertices, which are bilinear in fermion and antifermion creation and annihilation operators, and linear in boson creation and annihilation operators. The fermionantifermion operators generate a unitary Lie algebra, whose representations are fixed by a first order Casimir operator (corresponding to baryon number or charge). Eigenvectors and eigenvalues of the four-momentum operator are analyzed and exact solutions in the strong coupling limit are sketched. A simple model shows how the fine structure constant might be determined for the QED vertex.
\end{abstract}

Key words: point form relativistic quantum mechanics; antisymmetric representations of infinite unitary groups; semidirect sum of unitary with Heisenberg algebra

2000 Mathematics Subject Classification: 22D10; 81R10; 81T27

\section{Introduction}

With the exception of QCD and gravitational self couplings, all of the fundamental particle interaction Hamiltonians have the form of bilinears in fermion and antifermion creation and annihilation operators times terms linear in boson creation and annihilation operators. For example QED is a theory bilinear in electron and positron creation and annihilation operators and linear in photon creation and annihilation operators. The well-known nucleon-antinucleonmeson interactions are of this form as are the weak interactions. These interactions differ of course in the way the fermions are coupled to the bosons, including the way in which internal symmetries are incorporated. An exception is QCD, where due to the $S U(3)_{\text {color }}$ symmetry which generates gluon self coupling terms, the gluon sector is no longer linear in creation and annihilation operators. The other exception is the gravitational interaction; since gravitons carry energy and momentum, they also can couple to themselves.

If $a^{\dagger}, b^{\dagger}$ and $c^{\dagger}$ denote, respectively, fermion, antifermion and boson creation operators, the aformentioned interactions can all be written as $\left(a^{\dagger}+b\right)\left(a+b^{\dagger}\right)\left(c^{\dagger}+c\right)$, while the "kinetic energy" terms are of the form $a^{\dagger} a-b b^{\dagger}+c^{\dagger} c$. Written in this way, the fermion-antifermion bilinears $a^{\dagger} a$, $b b^{\dagger}, a^{\dagger} b^{\dagger}$, and $b a$ close to form a Lie algebra which, with indices attached to the creation and annihilation operators, is related to the group $U(2 N)$. Similarly the boson operators $c^{\dagger} c, c^{\dagger}$ and $c$ close to form a Lie algebra related to the semidirect sum of unitary with Heisenberg algebras. Then the aforementioned interactions can all be viewed as products of these two Lie algebras.

The goal of this paper is to exploit this Lie algebra structure to analyze eigensolutions of the four-momentum operator. The four-momentum operator $P^{\mu}$ will be written as the sum of free and interacting four-momentum operators $P^{\mu}=P^{\mu}(\mathrm{fr})+P^{\mu}(I)$, where the free four-momentum operator has the "relativistic kinetic energy" form of creation and annihilation operators, while the interacting four-momentum operator has the Lie algebra product structure. 
To guarantee the relativistic covariance of the theory, it is required that

$$
\begin{aligned}
& {\left[P^{\mu}, P^{\nu}\right]=0,} \\
& U_{\Lambda} P^{\mu} U_{\Lambda}^{-1}=\left(\Lambda^{-1}\right)_{\nu}^{\mu} P^{\nu},
\end{aligned}
$$

where $U_{\Lambda}$ is the unitary operator representing the Lorentz transformation $\Lambda$. These "point form" equations $[1]^{1}$, in which all of the interactions are in the four-momentum operator and the Lorentz transformations are kinematic, lead to the eigenvalue problem

$$
P^{\mu}\left|\Psi_{p}\right\rangle=p^{\mu}\left|\Psi_{p}\right\rangle
$$

where $p^{\mu}$ is the four-momentum eigenvalue and $\left|\Psi_{p}\right\rangle$ the eigenvector of the four-momentum operator, which is an element in a generalized fermion-antifermion-boson Fock space. The physical vacuum and physical bound and scattering states should then all arise as the appropriate solutions of the eigenvalue equation (3). How this might be done is sketched in the following sections.

\section{Vertex Interactions}

The free four-momentum operator is generated from irreducible representations of the Poincaré group $^{2}$. The positive mass, positive spin representations act on a representation space that can be written as the product of square integrable functions over the forward hyperboloid times a spin space, $L^{2}(S O(1,3) / S O(3)) \otimes V^{j}$, with the group action on states given by

$$
U_{\Lambda}|v, \sigma\rangle=\sum\left|\Lambda v, \sigma^{\prime}\right\rangle D_{\sigma^{\prime}, \sigma}^{j}\left(R_{W}\right), \quad U_{a}|v, \sigma\rangle=e^{i m v \cdot a}|v, \sigma\rangle,
$$

here $a$ is a four-vector space-time translation, $v$ is the four-velocity on the forward hyperboloid satisfying $v \cdot v=1$ and $\sigma$ is the relativistic spin projection ranging between $-j$ and $j$ and generating a basis in the $2 j+1$ dimensional spin space $V^{j} . m$ is a mass parameter that (along with the spin $j$ ) labels an irreducible representation; the usual four-momentum is defined by $p=m v . D^{j}(\cdot)$ in equation (4) is a matrix element of the rotation group, with its argument a Wigner rotation defined by $R_{W}=B^{-1}(\Lambda v) \Lambda B(v)$ [5].

From this irreducible representation it is possible to define many-particle creation and annihilation operators which satisfy the following (anti)commutation relations:

$$
\left[a_{i}, a_{i^{\prime}}^{\dagger}\right]_{+}=\delta_{i, i^{\prime}}, \quad\left[b_{i}, b_{i^{\prime}}^{\dagger}\right]_{+}=\delta_{i, i^{\prime}}, \quad\left[c_{k}, c_{k^{\prime}}^{\dagger}\right]_{-}=\delta_{k, k^{\prime}},
$$

the $a_{i}, b_{i}, c_{k}$ are respectively bare fermion, antifermion and boson annihilation operators and satisfy appropriate commutation or anticommutation relations with the associated creation operators. They all transform in the same way as one-particle states. For example, $U_{\Lambda} a_{v, \sigma}^{\dagger} U_{\Lambda}^{-1}=$ $\sum a_{\Lambda v, \sigma^{\prime}}^{\dagger} D_{\sigma^{\prime}, \sigma}^{j}\left(R_{W}\right)$. The subscripts on these operators denote whatever set of labels is inherited from the one-particle representations. Thus, if the fermions are labelled by $v$ and $\sigma$, the Kronecker delta on the right hand sides of equations (5) stands for $v_{0} \delta^{3}\left(v-v^{\prime}\right) \delta_{\sigma, \sigma^{\prime}}$. It should be noted that the three (anti)commutation relations are all dimensionless; that is, by using the four-velocity rather than the four-momentum, all quantities are dimensionless.

The free four-momentum operator can be written in terms of the bilinears as

$$
P^{\mu}(\mathrm{fr}):=m \sum \int d v v^{\mu}\left(a_{i}^{\dagger} a_{i}+b_{i}^{\dagger} b_{i}+\kappa c_{k}^{\dagger} c_{k}\right)
$$

\footnotetext{
${ }^{1}$ For a discussion of the various forms of dynamics, see for example [2].

${ }^{2}$ The irreducible representations of the Poincaré group are worked out in many texts; good examples of group theory texts are the texts by A.O. Barut and R. Rączka [3] and W. Tung [4].
} 
where, as stated, $i$ stands for the collection of variables which includes the four-velocity and other discrete variables such as spin projections or internal symmetry labels. $d v=\frac{d^{3} v}{v_{0}}$ is the Lorentz invariant measure over the four-velocity, which is common to all three types of particles. $k$ similarly always contains the four-velocity, along with other discrete variables that may differ from the fermionic variables. $\kappa$ is a dimensionless relative bare boson mass parameter which is determined from solutions of the eigenvalue equation for physical particles. Thus, the only quantity with a dimension that appears in equation (6) is the bare fermion mass $m$; its value is determined by relating a physical mass such as the nucleon mass to the dimensionless eigenvalue of the corresponding stable particle. Because of the transformation properties of the creation and annihilation operators inherited from the one-particle states, the free four-momentum operator as defined in equation (6) satisfies the point form equations (1) and (2).

The interacting four-momentum operator is generated from vertices, products of free field operators themselves made from creation and annihilation operators. If the vertex operator is denoted by $V(x)$, where $x$ is a space-time point, then the interacting four-momentum operator is obtained by integrating the vertex operator over the forward hyperboloid:

$$
P^{\mu}(I):=g \int d^{4} x \delta\left(x \cdot x-\tau^{2}\right) \theta\left(x_{0}\right) x^{\mu} V(x)=g \int d x x^{\mu} V(x),
$$

where $g$ is a coupling constant and the measure $d x$ is defined from equation (7). The vertex operator is required to be a scalar density under Poincaré transformations and have locality properties. That is,

$$
\begin{aligned}
& U_{a} V(x) U_{a}^{-1}=V(x+a), \\
& U_{\Lambda} V(x) U_{\Lambda}^{-1}=V(\Lambda x),
\end{aligned}
$$

$[V(x), V(y)]=0$ if $(x-y)^{2}$ is spacelike. Here $U_{a}=e^{-i P(\mathrm{fr}) \cdot a}$ is the free four-translation operator, with $P^{\mu}(\mathrm{fr})$ defined in equation (6).

Making use of the fact that if $x$ and $y$ are two time-like four-vectors with the same length $\tau$ so that their difference is space-like, it follows that $\left[P^{\mu}(I), P^{\nu}(I)\right]=0$. Also, from the Lorentz transformation properties of the vertex given in equation (9) it follows that the interacting four-momentum operator transforms as a four-vector. Thus $P^{\mu}(I)$ also satisfies the point form equations (1) and (2).

However, what is important for a relativistic theory is that the total four-momentum operator satisfy the point form equations. Making the four-translations in equation (8) infinitesimal gives $\left[P^{\nu}(\mathrm{fr}), P^{\mu}(I)\right]=\int d x x^{\mu} \frac{\partial}{\partial x_{\nu}} V(x)$ so that

$$
\begin{aligned}
& {\left[P^{\nu}(\mathrm{fr}), P^{\mu}(I)\right]-\left[P^{\mu}(\mathrm{fr}), P^{\nu}(I)\right]=g \int d x\left(x^{\mu} \frac{\partial}{\partial x_{\nu}}-x^{\nu} \frac{\partial}{\partial x_{\mu}}\right) V(x)=0,} \\
& {\left[P^{\mu}(\mathrm{fr})+P^{\mu}(I), P^{\nu}(\mathrm{fr})+P^{\nu}(I)\right]=\left[P^{\mu}, P^{\nu}\right]=0 .}
\end{aligned}
$$

Thus the total four-momentum operator also satisfies the point form equations.

As stated in the Introduction, the vertex is assumed to be bilinear in fermion-antifermion creation and annihilation operators, and linear in boson creation and annihilation operators. That is, $V(x) \sim\left(a^{\dagger}+b\right)\left(a+b^{\dagger}\right)\left(c+c^{\dagger}\right)$. For example, for electromagnetic interactions the vertex is the product of a local current operator times the photon field, $V(x)=J^{\mu}(x) A_{\mu}(x)$, where the current operator is a bilinear in fermion-antifermion creation and annihilation operators (for example electron and positron creation and annihilation operators) and the photon field is linear in photon creation and annihilation operators. Thus, the vertex operator has the structure of a $U(2 \infty)$ Lie algebra (which is analyzed in the next section) times a Heisenberg-like algebra arising from the boson part of the vertex. 


\section{$3 \quad$ Representation structure of $U(2 N)$}

The fermion-antifermion creation and annihilation operators appear in the four-momentum operator only in the combinations $a_{i}^{\dagger} a_{j}, b_{i} b_{j}^{\dagger}, a_{i}^{\dagger} b_{j}^{\dagger}$ and $b_{i} a_{j}$. To analyze the representation structure of such an algebra it is convenient to temporarily assume the indices are discrete and range over $N$ values. However, in the following sections the indices will not be restricted to finite values.

To see how the four bilinears are related to a unitary algebra, consider the following correspondence with fermionic operators $A_{\alpha}, A_{\beta}^{\dagger}$ ranging over $2 N$ values: $a_{i}^{\dagger} \rightarrow A_{i}^{\dagger}, a_{i} \rightarrow A_{i}$, $b_{i} \rightarrow A_{i+N}^{\dagger}, b_{i}^{\dagger} \rightarrow A_{i+N}$, where bilinears in the $A^{\prime}$ 's and $A^{\dagger}$ 's satisfy the unitary Lie algebra (over $2 N$ values) commutation relations

$$
\left[A_{\alpha}^{\dagger} A_{\beta}, A_{\mu}^{\dagger} A_{\nu}\right]=A_{\alpha}^{\dagger} A_{\nu} \delta_{\beta, \mu}-A_{\mu}^{\dagger} A_{\beta} \delta_{\alpha, \nu} .
$$

For example

$$
\left[b_{i} a_{j}, a_{k}^{\dagger} b_{l}^{\dagger}\right]=\left[A_{i+N}^{\dagger} A_{j}, A_{k}^{\dagger} A_{l+N}\right]=A_{i+N}^{\dagger} A_{l+N} \delta_{j, k}-A_{k}^{\dagger} A_{j} \delta_{i, l}=b_{i} b_{l}^{\dagger} \delta_{j, k}-a_{k}^{\dagger} a_{j} \delta_{i, l}
$$

as required. It should be noted that if the antifermion creation and annihilation operators are normal ordered, an extra term (central extension) appears in the commutation relations. To avoid such terms and keep the correspondence with the unitary algebra, the antifermion creation and annihilation terms are not normal ordered.

Define

$$
\mathcal{A}(X):=A_{\alpha}^{\dagger} X_{\alpha \beta} A_{\beta}, \quad 0 \leq \alpha, \beta \leq 2 N,
$$

then all fermionic terms in $P^{\mu}$ are of this form. For example, the free fermionic four-momentum operator can be written, up to a constant, as

$$
P_{F}^{\mu}(\mathrm{fr})=m \sum \int d v v^{\mu}\left(a_{i}^{\dagger} a_{i}-b_{i} b_{i}^{\dagger}\right)=m \sum \int \mathcal{A}\left(E^{\mu}\right), \quad E^{\mu}=\operatorname{diag}\left(v^{\mu},-v^{\mu}\right),
$$

where in this expression continuous variables have been included.

Given the unitary algebra of $U(2 N)$, representations can be given in a variety of ways. In the Gelfand-Zetlin scheme ${ }^{3}$, in which irreducible representations of the unitary groups are labelled by nonnegative arrays of integers, the relevant fermionic-antifermionic representations are all the antisymmetric representations, written $(1, \ldots, 1,0, \ldots, 0)$, of length $2 N$, with the identity representations given by all zeroes or all ones. The antisymmetric Fock space is the direct sum of all these irreducible representation spaces, and is of dimension $2^{2 N}$.

When there are no internal symmetries, a more convenient way of labelling the irreducible representations is with a "baryon number" (or charge) operator. Define $\mathcal{B}:=\sum \int\left(a_{i}^{\dagger} a_{i}+\right.$ $\left.b_{i} b_{i}^{\dagger}\right)=\sum \int \mathcal{A}(I)$, a first order Casimir operator with eigenvalues from $-N$ to $+N$. Each integer eigenvalue corresponds to a given irreducible representation. For example the $\mathcal{B}=0$ sector corresponds in the Gelfand-Zetlin notation to arrays with equal numbers of zeroes and ones.

For each baryon number sector there is a cyclic vector which is annihilated by the lowering operators $b a$, and the raising operators then generate all the possible states, up to a maximum value, at which point the raising operator annihilates. Such a construction begins with the fermion-antifermion Fock vacuum, $\left|0_{F}\right\rangle=|0\rangle|0\rangle$, the tensor product of fermion and anti-fermion Fock vacuum.

Starting with baryon number zero, the fermion-antifermion cyclic vector is defined by $a_{i}\left|0_{F}\right\rangle=$ $b_{i}\left|0_{F}\right\rangle=0$ and hence satisfies $b_{i} a_{j}\left|0_{F}\right\rangle=0, \mathcal{B}\left|0_{F}\right\rangle=0$. States are generated from the vacuum

\footnotetext{
${ }^{3} \mathrm{~A}$ good reference for the $U(N)$ groups, including the Gelfand-Zetlin labeling scheme, is given in [6].
} 
by products of raising operators $\left|i_{1} j_{1} \cdots\right\rangle:=a_{i_{1}}^{\dagger} b_{j_{1}}^{\dagger} \cdots\left|0_{F}\right\rangle$. The vacuum carries the identity representation for each $U(N)$ subalgebra, whereas the states $|i, j\rangle:=a_{i}^{\dagger} b_{j}^{\dagger}\left|0_{F}\right\rangle$ carry the tensor product of the fundamental representation of the $U(N)$ algebras.

Similarly for baryon number one, a cyclic vector is given by $|0\rangle_{1}:=a_{i}^{\dagger}\left|0_{F}\right\rangle$, with $b_{i} a_{j}|0\rangle_{1}=0$, $\mathcal{B}|0\rangle_{1}=|0\rangle_{1}$. Such a cyclic vector carries the fundamental representation of the $a^{\dagger} a$ algebra, and the identity representation of the $b^{\dagger} b$ algebra. A basis in the baryon number one irrep space is again given by products of "bare fermion-antifermion" pairs $\left|i_{1} j_{1} \cdots\right\rangle_{1}=a_{i_{1}}^{\dagger} b_{j_{1}}^{\dagger} \cdots|0\rangle_{1}$. The dimension of this irrep space is smaller than the baryon zero irrep space, because there are fewer "fermion-antifermion" pairs that are available to span the space.

Continuing in this way there is finally the baryon $N$ irrep space, a one dimensional space whose only state is the cyclic vector itself, $|0\rangle_{N}:=a_{1}^{\dagger} \cdots a_{N}^{\dagger}\left|0_{F}\right\rangle, \mathcal{B}|0\rangle_{N}=N|0\rangle_{N}$. The other one dimensional irrep space is the baryon number $-N$ space, defined by replacing the fermion creation operators with anti-fermion creation operators. These representations do not exist when $N \rightarrow \infty$, which means they do not occur when continuous variables are included.

\section{Eigenvalue structure of the four-momentum operator}

If the definition, equation (10) is substituted into equation (7), the interacting four-momentum operator can be written as

$$
\begin{aligned}
& P^{\mu}(I)=g \sum \int\left(\mathcal{A}\left(X_{k}^{\mu}\right) c_{k}+\mathcal{A}\left(X_{k}^{\mu}\right)^{\dagger} c_{k}^{\dagger}\right) \\
& {\left[X_{k}^{\mu}, X_{l}^{\nu}\right]=0} \\
& {\left[X_{k}^{\mu},\left(X_{l}^{\mu}\right)^{\dagger}\right]=0 .}
\end{aligned}
$$

Equations (13) and (14) follow from the fact that the components of the interacting fourmomentum operator commute, and that the four-momentum operator is hermitian. Equation (14) implies that the $X$ 's are normal operators, while equation (13) implies that they can all be simultaneously diagonalized.

The $\left(X_{k}^{\mu}\right)_{\alpha \beta}$ are obtained from a given vertex; for example for the pseudoscalar coupling of a meson to nucleons and antinucleons, two of the four terms in equation (12) are

$$
\begin{aligned}
& \left(X_{k}^{\mu}\right)_{i j}=F^{\mu}\left(v_{1}-v_{2}-v\right) \bar{u}\left(v_{1}, \sigma_{1}\right) \gamma_{5} u\left(v_{2}\right), \\
& \left(X_{k}^{\mu}\right)_{i+N, j}=F^{\mu}\left(-v_{1}-v_{2}-v\right) \bar{v}\left(v_{1}, \sigma_{1}\right) \gamma_{5} u\left(v_{2}\right), \\
& F^{\mu}(u):=\int d^{4} y \delta(y \cdot y-1) \theta\left(y_{0}\right) y^{\mu} e^{i u \cdot y} .
\end{aligned}
$$

Since all the quantities in equation (12) except the coupling constant $g$ are dimensionless, $g$ has the dimensions of mass. If such a coupling constant is written as $g=m \alpha$, where $m$ is the mass factor multiplying the free four-momentum operator, equation (6), then $\alpha$ is a dimensionless coupling constant. In the following the mass factor will be dropped, so that the four-momentum operator is dimensionless.

Now the reason for writing the interacting four-momentum operator in the form (12), is to solve the eigenvalue problem (3). If the one-particle eigenvalue problem can be solved, the mass value of the eigenvalue will be dimensionless. To connect it with a physical mass, the mass factor must be chosen so that the physical mass equals the factor $m$ times the dimensionless eigenvalue mass; in this way the mass factor multiplying both the free and interacting four-momentum operators is fixed. 
Combining the expressions for the terms in the four-momentum operator given in equations (6), (12) the eigenvalue problem (3) to be solved can be written as

$$
\left(P_{F}^{\mu}(\mathrm{fr})+\sum \int d v\left(\kappa v^{\mu} c_{k}^{\dagger} c_{k}+\alpha \mathcal{A}\left(X_{k}^{\mu}\right) c_{k}+\alpha \mathcal{A}\left(X_{k}^{\mu}\right)^{\dagger} c_{k}^{\dagger}\right)\right)\left|\Psi_{\lambda}\right\rangle=\lambda^{\mu}\left|\Psi_{\lambda}\right\rangle
$$

where the first term in equation (15) is the free fermion-antifermion four-momentum operator (11), the second term is the boson free four-momentum operator, with the factor $\kappa$ the dimensionless ratio of physical meson mass to physical fermion mass, and the third term is the interaction coupling bilinear fermions to the mesons, with a strength given by the dimensionless coupling constant $\alpha$. The point eigenvalues will give the masses of the stable particles, while the continuous part of the spectrum gives the scattering states.

Since the four-momentum operator transforms as a four-vector under Lorentz transformations, Lorentz covariance can be used to write $\lambda^{\mu}=(\lambda, 0,0,0)$. Then the eigenvector equation can be rewritten as

$$
P_{F}^{0}(\mathrm{fr})+\sum \int d v\left(\kappa v^{0} c_{k}^{\dagger} c_{k}+\alpha \mathcal{A}\left(X_{k}^{0}\right) c_{k}+\alpha \mathcal{A}\left(X_{k}^{0}\right)^{\dagger} c_{k}^{\dagger}\right)\left|\Psi_{\lambda}\right\rangle=\lambda\left|\Psi_{\lambda}\right\rangle
$$

There is also a corresponding eigenvector equation for the space components of the four-momentum operator, for which the eigenvalue is zero.

Next consider an automorphism on the Heisenberg Lie algebra, with transformation

$$
c_{k}=C_{k}-\frac{\alpha \mathcal{A}\left(X_{k}^{0}\right)^{\dagger}}{\kappa v^{0}}, \quad c_{k}^{\dagger}=C_{k}^{\dagger}-\frac{\alpha \mathcal{A}\left(X_{k}^{0}\right)}{\kappa v^{0}} .
$$

This will be an automorphism if the $C$ 's satisfy the correct commutation relations,

$$
\begin{aligned}
& {\left[C_{k}, C_{k^{\prime}}^{\dagger}\right]=\left[c_{k}+\frac{\alpha \mathcal{A}\left(X_{k}^{0}\right)^{\dagger}}{\kappa v^{0}}, c_{k^{\prime}}+\frac{\alpha \mathcal{A}\left(X_{k^{\prime}}^{0}\right)}{\kappa v^{0}}\right]=\delta_{k, k^{\prime}},} \\
& {\left[C_{k}^{\dagger}, C_{k^{\prime}}^{\dagger}\right]=\left[c_{k}^{\dagger}+\frac{\alpha \mathcal{A}\left(X_{k}^{0}\right)}{\kappa v^{0}}, c_{k^{\prime}}^{\dagger}+\frac{\alpha \mathcal{A}\left(X_{k^{\prime}}^{0}\right)}{\kappa v^{0}}\right]=0 .}
\end{aligned}
$$

These commutation relations follow since the $\mathcal{A}(X)$ 's commute among themselves, equations (13).

Since the $C$ 's also satisfy boson commutation relations, the eigenvalue equation can be written in terms of them:

$$
\left(P_{F}^{0}(\mathrm{fr})-\alpha^{2} \sum \int d v \frac{1}{\kappa v^{0}} \mathcal{A}\left(X_{k}^{0}\right) \mathcal{A}\left(X_{k}^{0}\right)^{\dagger}+\sum \int d v \kappa v^{0} C_{k}^{\dagger} C_{k}\right)\left|\Psi_{\lambda}\right\rangle=\lambda\left|\Psi_{\lambda}\right\rangle .
$$

If $\alpha=0$ then the ground state solution is the usual Fock space solution; but the above operators are all positive, so there can never be a Fock space solution for $\alpha \neq 0$, that is, when the physical vacuum is in the Fock space. The goal is to look for solutions of equation (16) that lead to a suitable choice for the physical vacuum.

\section{$5 \quad$ A simple model}

To motivate the kinds of considerations that go into getting a physical vacuum, consider a simple model in which $N$ is finite and the baryon number is $N$. Then it is straightforward to get exact solutions to the eigenvalue problem because the fermion-antifermion sector is one-dimensional.

To get these solutions, it is simplest to realize the boson sector in Bargmann variables, namely, $c_{k}^{\dagger} \rightarrow z_{k}, c_{k} \rightarrow \frac{\partial}{\partial z_{k}}$ on a holomorphic Hilbert space [7]. Then the Hamiltonian and eigenfunctions, eigenvalues, are

$$
H=\sum e_{i}\left(a_{i}^{\dagger} a_{i}+b_{i}^{\dagger} b_{i}+z_{i} \frac{\partial}{\partial z_{i}}\right)+\alpha \sum \mathcal{A}\left(X_{i}^{0}\right) \frac{\partial}{\partial z_{i}}+\mathcal{A}\left(X_{i}^{0}\right)^{\dagger} z_{i}
$$




$$
\begin{aligned}
= & \sum e_{i}+\sum \mathcal{A}(E)+\sum e_{i}\left(z_{i}+\frac{\alpha \mathcal{A}\left(X_{i}^{0}\right)}{e_{i}}\right)\left(\frac{\partial}{\partial z_{i}}+\frac{\alpha \mathcal{A}\left(X_{i}^{0}\right)^{\dagger}}{e_{i}}\right) \\
& -\alpha^{2} \sum \mathcal{A}\left(X_{i}^{0}\right) \mathcal{A}\left(X_{i}^{0}\right)^{\dagger}, \\
\left|\Psi_{n}\right\rangle= & \prod\left(e_{k} z_{k}+\frac{\alpha \mathcal{A}\left(X_{k}^{0}\right)}{e_{k}}\right)^{n_{k}} e^{-\alpha \sum \frac{\mathcal{A}\left(X_{i}^{0}\right) z_{i}}{e_{i}}} a_{1}^{\dagger} \cdots a_{N}^{\dagger}|0\rangle, \\
\lambda_{n}= & \sum\left(e_{i}+n_{i} e_{i}-\frac{\alpha^{2}\left|Y_{i}^{0}\right|^{2}}{e_{i}}\right), \\
\lambda_{\min }= & \sum e_{i}-\alpha^{2} \sum \frac{\left|Y_{i}^{0}\right|^{2}}{e_{i}} \neq 0 .
\end{aligned}
$$

Here $e_{i}$ is the discretized energy, $\left(Y_{i}^{0}\right)=\sum\left(\left(X_{i}^{0}\right)_{j j}+\left(X_{i}^{0}\right)_{j+N, j+N}\right)$, and $\kappa$ has been set to one. The minimum eigenvalue has a positive term coming from the free "relativistic energy", balanced by a negative term, coming from the interaction between fermions and bosons.

This simple model shows that the minimum eigenvalue expresed by (18) is not zero. But the vacuum by definition has zero energy. As seen in (18) only a definite value of the coupling constant will satisfy such a requirement; that is, $H\left|\Psi_{\min }\right\rangle=0$ determines the value of the coupling constant.

Now remove the $N$ finite restriction, replace the above Hamiltonian with the electromagnetic Hamiltonian and replace the baryon number operator with the charge operator in the charge zero sector. Since the free photon four-momentum operator has no $\kappa$ factor (since photons are massless), the vacuum solution of $H$, namely $H|\Omega\rangle=0$, should fix the value of $\alpha$, which gives the fine structure constant. Note that unlike the case of a Hamiltonian operator only, where it is always possible to add an arbitrary constant to the Hamiltonian and hence shift the energy by an arbitrary amount, in the case of the four-momentum operator this is not possible without destroying the Lorentz covariance of the operator.

A more interesting example is the above model in the $N=1$, baryon number 0 sector. Then the Hamiltonian and eigenfunctions for a simple choice of $X$ can be written as

$$
\begin{aligned}
& H=e\left(a^{\dagger} a+b^{\dagger} b\right)+z \frac{\partial}{\partial z}+\alpha\left(a^{\dagger} b^{\dagger}+b a\right)\left(\frac{\partial}{\partial z}+z\right), \\
& \left|\Psi_{n}\right\rangle=f_{1}(z)|0\rangle+f_{2}(z) a^{\dagger} b^{\dagger}|0\rangle .
\end{aligned}
$$

The eigenvalue problem generates a matrix differential equation

$$
\begin{aligned}
& z \frac{\partial f_{1}}{\partial z}+\alpha \frac{\partial f_{2}}{\partial z}=\lambda f_{1}-\alpha z f_{2}, \\
& z \frac{\partial f_{2}}{\partial z}+\alpha \frac{\partial f_{1}}{\partial z}=(\lambda-2 e) f_{2}-\alpha z f_{1}
\end{aligned}
$$

which can be rewritten as

$$
\begin{aligned}
& (z+\alpha) f_{+}^{\prime}=(\lambda-e-\alpha z) f_{+}+e f_{-}, \\
& (z-\alpha) f_{-}^{\prime}=(\lambda-e+\alpha z) f_{-}+e f_{+}
\end{aligned}
$$

with $f_{ \pm}:=f_{1} \pm f_{2}, f_{ \pm}$holomorphic. If $e$ is zero the solution has a structure given by expression (17). However, as far as the "vacuum" structure is concerned, $e$ not zero is the interesting case.

\section{Conclusion}

In the point form of relativistic quantum mechanics all interactions are in the four-momentum operator, and Lorentz transformations are kinematic (that is, Lorentz generators are free of 
interactions). Since the four-momentum operator can be written as bilinear in fermion, antifermion creation and annihilation operators, a Lie algebra is generated, which is isomorphic to the Lie algebra of the unitary group. Further, the representations of interest are the antisymmetric representations, and can be labelled by integers, the eigenvalues of the baryon number operator. Similarly the bosonic parts of the four-momentum operator are elements of a Lie algebra, related to the semidirect sum of a unitary algebra with the Heisenberg algebra.

Via the automorphism defined in Section 4, the four-momentum operator can be rewritten in such a way as to exhibit properties of its eigenvalue structure. Though it is not discussed in this paper, a strong coupling approximation of the four-momentum operator, in which the fermion free relativistic energy is neglected, can be solved exactly and has a structure similar to the solutions given in equation (17).

Several simple models show a balance of the minimum eigenvalue between fermionic relativistic energy and fermion-boson coupling terms; this leads to the possibility that the fine structure constant may be determined from requirements on the physical vacuum. However much remains to be done to connect the simple models discussed here with genuine infinite degree of freedom systems.

[1] Klink W.H., Point form relativistic quantum mechanics and electromagnetic form factors, Phys. Rev. C, 1998, V.58, 3587-3604.

[2] Keister B.D., Polyzou W.N., Relativistic Hamiltonian dynamics in nuclear and particle physics, Advances Nuclear Physics, Editors J.W. Negele and E.W. Vogt, New York, Plenum, 1991, V.20, 225-479.

[3] Barut A.O., Rạczka R., Theory of group representations and applications, Warsaw, Polish Scientific Publishers, 1977.

[4] Tung W., Group theory in physics, Singapore, World Scientific, 1985.

[5] Klink W.H., Relativistic simultaneously coupled multiparticle states, Phys. Rev. C, 1998, V.58, 3617-3626.

[6] Zhelobenko D.P., Compact Lie groups and their representations, Providence, R.I. American Mathematical Society, 1973.

[7] Bargmann V., On a Hilbert space of analytic functions and an associated integral transform, Comm. Pure Appl. Math., 1961, V.16, 187-214. 